\title{
NUDT15 R139C Variants Increase the Risk of Azathioprine-Induced Leukopenia in Chinese Autoimmune Patients
}

\author{
Xiang Fei ${ }^{1,2}$, Qing Shu ${ }^{1}$, Huaijun Zhu ${ }^{1}$, Bingzhu Hua ${ }^{3}$, Shiying Wang ${ }^{3}$, Ling Guo ${ }^{4}$, \\ Yun Fang ${ }^{1 *}$ and Weihong $\mathrm{Ge}^{1 *}$
}

${ }^{1}$ Department of Pharmacy, Nanjing Drum Tower Hospital, Nanjing, China, ${ }^{2}$ School of Basic Medicine and Clinical Pharmacy, China Pharmaceutical University, Nanjing, China, ${ }^{3}$ Department of Rheumatology and Immunology, Nanjing Drum Tower Hospital, Nanjing, China, ${ }^{4}$ Nanjing Drum Tower Hospital, Clinical College of Traditional Chinese and Western Medicine, Nanjing University of Chinese Medicine, Nanjing, China

OPEN ACCESS

Edited by:

Chonlaphat Sukasem, Mahidol University, Thailand

Reviewed by:

Julio Benitez,

Universidad de Extremadura, Spain

Suda Vannaprasaht

Faculty of Medicine, Khon Kaen

University, Thailand

Murray Barclay,

Canterbury District Health Board,

New Zealand

*Correspondence:

Yun Fang

njglfy@163.com

Weihong Ge

6221230@sina.com

Specialty section:

This article was submitted to

Pharmacogenetics and

Pharmacogenomics,

a section of the journal

Frontiers in Pharmacology

Received: 15 November 2017

Accepted: 19 April 2018

Published: 07 May 2018

Citation:

Fei X, Shu Q, Zhu H, Hua B, Wang S,

Guo L, Fang $Y$ and Ge W (2018)

NUDT15 R139C Variants Increase the

Risk of Azathioprine-Induced

Leukopenia in Chinese Autoimmune

Patients. Front. Pharmacol. 9:460.

doi: 10.3389/fphar.2018.00460
The aim of this study was to investigate the influence of NUDT15 R139C, thiopurine S-methyltransferase (TPMT), and 6-TGN on azathioprine (AZA) induced leukopenia in Chinese autoimmune patients. Among 87 enrolled patients, 23 (26.4\%) had leukopenia. The NUDT15 R139C variant was associated with leukopenia $\left(p=1.86 \times 10^{-7}\right.$; OR: 7.59; 95\% Cl: 3.16-18.21). However, TPMT genotype was not shown to be correlated with the incidence of leukopenia $(p=0.95)$. There was no significant difference of 6 -TGN concentration between patients with or without leukopenia $(p=0.15)$ and no association was found in patients with NUDT15 R139C variants alleles $(p=0.62)$. Finally, we found that the range of 6-TGN concentrations in autoimmune diseases was much lower than the established 6-TGN monitoring range for inflammatory bowel diseases. Therefore, the variant of NUDT15 R139C is strongly associated with AZA-induced leukopenia in Chinese patients with various autoimmune diseases such as systemic lupus erythematosus, Sjögren's syndrome, etc.

Keywords: azathioprine, NUDT15 R139C, leukopenia, Chinese, autoimmune patients

\section{INTRODUCTION}

Azathioprine (AZA) is a thiopurine prodrug commonly used as an immunosuppressive agent in the treatment of inflammatory bowel disease (IBD), systemic lupus erythematosus (SLE), Sjögren's syndrome (SS), and other autoimmune diseases (Bertsias et al., 2008; Ramos-Casals et al., 2010; Timmer et al., 2012, 2016; Janssens et al., 2013; Okon and Werth, 2013). AZA is relatively safe for clinical use, though several studies have reported up to $50 \%$ of patients discontinued AZA during long-term therapy, mainly due to the adverse drug reactions (ADRs) (de Jong et al., 2003; Jharap et al., 2010). Myelosuppression, one of the AZA-induced life-threatening adverse events, occurred in 3-17\% of these patients (Boonsrirat et al., 2008; Ngo et al., 2011).

As a prodrug, AZA is nonenzymatically broken down to 6-mercaptopurine (6-MP), and then 6-MP is converted to 6 -thioguanine nucleotides (6-TGN) or various other metabolites by a number of enzymes (Teml et al., 2007; Moon and Loftus, 2016). The predominant active metabolites 6-TGN cause cytotoxicity by interfering with de novo purine biosynthesis and modification of DNA structure after their incorporation into nucleic acids (Somerville et al., 2003). Finally, part 
of 6-TGN is hydrolysed to inactive metabolites by nucleoside diphosphate-linked moiety X-type motif 15 (NUDT15), which is one member of the nudix hydrolase enzyme family (Carter et al., 2015).

In this process, thiopurine S-methyltransferase (TPMT) metabolizes AZA to inactive molecules, and variant TPMT alleles (slow metabolizer) are associated with AZA-induced leukopenia with an elevation of 6-TGN level (Hiratsuka et al., 2000). The association between AZA-induced leukopenia and TPMT mutations is well-established. TPMT gene testing before AZA exposure is recommended by the US Food and Drug Administration (FDA) to predict adverse events and guide selecting doses of AZA (Relling et al., 2013). However, increasing studies found that the frequency of TPMT mutation is considerably lower in Chinese than in Caucasians, with the lowest frequencies observed in Chinese (about 0.9\%; Fangbin et al., 2012; Zhu and Cao, 2012) compared with a higher incidence of AZA-induced leukopenia (27-41.3\%; Connell et al., 1993). A recent study found that NUDT15 R139C was strongly associated with AZA-induced leukopenia in Koreans (Yang et al., 2014). Additionally, (Zhu et al., 2016) confirmed the association of NUDT15 R139C with early leukopenia in Chinese IBD patients commencing AZA treatment. These findings suggested that NUDT15 R139C is a potential genetic factor that is responsible for AZA-induced leukopenia in East Asian populations. Azathioprine is more widely used in various autoimmune diseases like SLE and SS compared to IBD. However, the association of NUDT15 R139C variants with AZA-induced leukopenia had not been reported in Chinese autoimmune diseases.

Moreover, the clinical efficacy of AZA had been reported to be correlated with erythrocyte levels of 6-TGN (Cuffari et al., 1996; Osterman et al., 2006). An improved clinical response to AZA has been reported in IBD patients when the concentration of 6-TGN was $235 \mathrm{pmol} / 8 \times 10^{8} \mathrm{RBC}$ or higher (Dubinsky et al., 2000; Teml et al., 2007; Hanai et al., 2010). However, leukopenia occurred when higher 6-TGN levels $\left(450 \mathrm{pmol} / 8 \times 10^{8} \mathrm{RBC}\right)$ were achieved (Dubinsky et al., 2000). Another study in SLE patients found that clinical response can occur at lower 6-TGN levels than the target range established for IBD (Osterman et al., 2006). The distribution of 6-TGN levels in Chinese autoimmune diseases is still unknown.

Our study was aimed to investigate NUDT15 R139C, $T_{P M T} 3 C, 6$-TGN levels and explore their influence on AZAinduced leukopenia in Chinese autoimmune diseases (except IBD), retrospectively.

\section{MATERIALS AND METHODS}

\section{Patient Recruitment}

A total of 87 patients with autoimmune diseases with AZA therapy for more than 2 months were recruited in the Drum Tower Hospital affiliated Nanjing University Medical School from Sep 1st 2016 to Apr 1st 2018. These patients exhibited various autoimmune diseases including SLE, SS, dermatomyositis (DM), and others. All subjects were Chinese, and their clinical characteristics are shown in Table 1. Exclusion
TABLE 1 | Baseline characteristics of subjects in this study.

\begin{tabular}{lccc}
\hline \multirow{2}{*}{ Characteristics } & \multicolumn{2}{c}{ Patient } & p \\
\cline { 2 - 3 } & Leukopenia & Controls & \\
\hline No. of subjects (\%) & $23(26.4 \%)$ & $64(73.6 \%)$ & - \\
Female (\%) & $23(100 \%)$ & $56(87.5 \%)$ & 0.001 \\
Age (years) & $37.0 \pm 9.94$ & $35.4 \pm 10.25$ & 0.636 \\
AZA dose (mg/day) & $58.3 \pm 19.17$ & $55.8 \pm 16.13$ & 0.285 \\
WBC_0W & $5.5 \pm 1.02$ & $5.9 \pm 1.12$ & 0.197 \\
NEU_0W & $3.9 \pm 1.34$ & $4.3 \pm 1.57$ & 0.068 \\
DISEASE & & & \\
SLE & 13 & 30 & \\
SS & 6 & 15 & \\
Vasculitis & 2 & 5 & \\
Scleroderma & 2 & 3 & \\
DM & 0 & 5 & \\
CTD & 0 & 3 & \\
IgG4-RD & 0 & 2 & \\
AlH & 0 & 1 &
\end{tabular}

OTHER ADVERSE EVENTS

Neutropenia

Severe hair loss

$10(43.5 \%)$

$\mathrm{O}(0 \%)$

$<0.001$

Vomiting

$2(8.7 \%)$

$\mathrm{O}(0 \%)$

$<0.001$

Controls, the patients without leukopenia; No., number of; AZA, azathioprine; WBC OW, the white blood cell counts at the initial of azathioprine treatment; NEU_Ow, neutrophil counts at the initional of azathioprine treatment; SLE, systemic lupus erythematosus; SS, Sjögren's syndrome; DM, dermatomyositis; CTD, connective tissue disease; IgG4-RD, IgG4 related diseases; $\mathrm{AlH}$, autoimmune hepatitis.

criteria included patients who were concomitantly treated with blood transfusion or other immunosuppressants that could result in leukopenia such as cyclosporine or tacrolimus (FK506), or treatments potentially interfering with AZA metabolism including allopurinol, 5-aminosalicylates and diuretics. Moreover, insufficient function of heart, liver, or kidney and suspected infection were excluded. The clinical data of these patients were analyzed from medical records, mainly including age, sex, body weight, combination of drugs, erythrocyte sedimentation rate (ESR), white blood count (WBC), red blood count (RBC), neutrophil count (NEU), platelet (PLT), alanine aminotransferase (ALT), and aspartate transaminase (AST), with a specific focus on leukopenia. This study was conducted in accordance with the ethical guidelines and approved by the research ethics committee of Drum Tower Hospital affiliated Nanjing University Medical School, Nanjing, China (2017-101-01). All methods were carried out in accordance with the approved guidelines.

\section{AZA Treatment and Adverse Events}

An initial dose of AZA at $50 \mathrm{mg}$ per day was commonly prescribed and adverse events were checked at 7-14 days from the beginning of medication. Routine blood counts were required once a week in the first month, then twice a month until 12 weeks. Two milliliters of venous blood samples (EDTA anticoagulation) were obtained at least 8 weeks after administration of AZA or 
at the time when adverse events occurred for erythrocyte 6TGN concentration measurement and gene testing. If patients developed any adverse drug events, the suspicious medication were stopped immediately and subsequent treatments were started by the responsible physician on a case-by-case basis.

AZA-induced leukopenia was defined as WBC of $<3.5 \times$ $10^{9} /$ L. Leukopenia before 8 weeks, and after 8 weeks were defined as early and late leukopenia, respectively. Neutropenia was defined as NEU of $<1.8 \times 10^{9} / \mathrm{L}$. Severe hair loss was defined as objective hair loss that patients may need to wear wigs or needed a few months to recover, and hepatotoxicity was regarded as ALT or AST >2-fold the upper normal limit without other causes. Gastrointestinal discomfort including nausea and vomiting.

The rheumatology specialists would confirmed the adverse events caused by AZA after carefully consideration. In order to rule out other causes, diagnostic criteria of leukopenia induced by AZA are as follows. Firstly, the initial WBC of enrolled patients is normal $\left(\mathrm{WBC}>3.5 \times 10^{9} / \mathrm{L}\right)$. Secondly, the time point of leukopenia happened is consistent with the acute phase of leukopenia caused by AZA (12 weeks). Thirdly, after withdraw of the AZA, the WBC of these patients who developed leukopenia would improve.

\section{Gene Analysis}

Total genomic DNA was isolated from peripheral leucocytes by a DNA extraction kit purchased from Promega (Madison, WI, USA). Genotyping for NUDT15 R139C (rs116855232) and $T P M T^{*} 3 C$ (rs1142345) were performed using Custom TaqMan ${ }^{\circledR}$ SNP genotyping assays (ID: C_154823200_10 and C__19567_20; Life Technologies, Carlsbad, CA, USA) in accordance with manufacture's information.

PCR was performed according to the manufacturer's instructions provided by Thermo Fisher Scientific. The PCR thermal cycling was as follows: initial denaturing at $92^{\circ} \mathrm{C}$ for 10 s followed by 50 cycles of $15 \mathrm{~s}$ at $92^{\circ} \mathrm{C}$ and $90 \mathrm{~s}$ at $60^{\circ} \mathrm{C}$ for annealing and extension. Thermal cycling was performed using a LightCycler 480 system (Roche Diagnostics, Switzerland). Each 96-well-plate contained 87 samples of an unknown genotype and 2 reaction mixtures containing the reagents, but no DNA (quality control). The no template controls were necessary for the Sequence Detection System (SDS) signal processing, as outlined in the TaqMan Allelic Discrimination Guide. The genotypes were determined visually based on the dye component fluorescent emission data depicted in the X-Y scatter-plot of the SDS software.

\section{Blood Concentration Measurement of 6-TGN}

The 6-TGN concentrations in erythrocyte were measured by high performance liquid chromatography, as previously described (Dervieux and Boulieu, 1998).

\section{Hardy-Weinberg Equilibrium (HWE)}

HWE analysis was performed on the research subjects by comparing the detected distribution of allele frequencies with the theoretical distribution estimated on the basis of the SNP allelic frequencies. $p>0.05$ (Chi-squared statistics) was considered to indicate equilibrium.

\section{Statistic Analysis}

Statistic analysis and calculations were performed by SPSS 20.0 (SPSS Inc., Chicago, IL, USA) and Prism 6 (Graph Pad Software, La Jolla, CA, USA). Data for continuous variables were expressed as the mean $\pm S D$, and those for categorized variables were expressed as frequencies. A one-sample KolmogorovSmirnov test was used to evaluate the normal distribution of 6-TGN metabolite concentrations. Categorical variables were compared using method $\chi^{2}$ or Fisher's test. A non-parametric Kruskal-Wallis H-test was used to evaluate the difference among independent groups. The sensitivity and specificity of NUDT15 $R 139 \mathrm{C}$ for detecting leukopenia and early leukopenia were calculated using the receiver operating characteristic curve (ROC). SHEsis (http://analysis.bio-x.cn/myAnalysis.php) was used to determine the deviation from the Hardy-Weinberg equilibrium. $p<0.05$ was regarded as statistically significant.

\section{RESULTS}

\section{Patient Characteristics}

A total of 98 consecutive patients were enrolled in this study. Among them, 11 patients were excluded from this study by the following reasons: refusal to participate in this study $(n=5)$; taking immunosuppressant concomitantly with AZA $(n=3)$; incomplete data for AZA treatment $(n=3)$. A total of 87 patients (79 females and 8 males) were analyzed in the present study. The percentage of females was much higher than males because females are more susceptible to autoimmune diseases like SLE, SS. Patients exhibated various autoimmune diseases as follows, SLE $(n=43)$, SS $(n=21)$, vasculitis $(n=7)$, scleroderma $(n=5)$, DM $(n=5)$, connective tissue disease (CTD) $(n=3)$, IgG4 related diseases $(n=2)$, and autoimmune hepatitis (AIH) $(n=1)$. The baseline characteristics of these patients are summarized in Table 1. Leukopenia was observed in 23 patients (26.4\%), 2 of them also experienced severe hair loss and 10 of them developed neutropenia during the follow-up visit.

The percentage of females who had severe hair loss and neutropenia was significantly higher than males $(p=0.001, p$ $<0.001$, respectively). No significant differences were observed in the age, AZA doses, initial WBC and NEU counts between individuals with or without leukopenia (controls) $(p>0.05$; Table 1).

NUDT15 R139C and TPMT*3C genotype distributions were in Hardy-Weinberg equilibrium ( $p=0.50$ and 0.85 respectively). Out of the 87 subjects, 59 subjects were NUDT15 R139C wild type (CC), which is nomal metabolizer (67.8\%) and 27 subjects were NUDT15 R139C heterozygote (CT) (31.0\%) while only one carried homozygote (TT) (1.1\%) which is poor metabolizer. The mutant allelic T frequency of NUDT15 R139C was $16.7 \%$ (29/174). In the same cohort, 83 patients (95.4\%) were wild type (TT) for $T P M T^{*} 3 C$ and 4 subjects $(4.6 \%)$ were $T P M T^{*} 3 C$ heterozygote (TC), the allelic C frequency of TPMT* $3 C$ was $2.3 \%$ (Table 2). 


\section{Association of NUDT15 R139C and TPMT*3C Genotype With Leukopenia}

Of the 59 patients with wild type NUDT15 R139C (CC), only $5(8.5 \%)$ suffered from leukopenia. Of the 27 patients with heterozygous NUDT15 R139C (CT), 17 patients $(63.0 \%)$ developed leukopenia and one patient with the homozygotes (TT) (100\%) suffered leukopenia and severe hair loss. The association of NUDT15 R139C with AZA-induced leukopenia was significant $\left(p=1.86 \times 10^{-7}\right)$. Compared with the wild type (CC), patients carrying variant allele T $(\mathrm{CT}+\mathrm{TT})$ had much higher risk in developing leukopenia $\left(p=1.79 \times 10^{-7}\right.$; OR $=7.59 ; 95 \%$; CI, 3.16-18.21; Table 3). For the early and late phase of leukopenia, the influence of mutant allele $\mathrm{T}$ was significantly different, with OR and 95\% CI as 8.85 (3.6421.53), 3.93 (0.37-41.39), respectively (Table 4). NUDT15 R139C genotypes were significantly associated with early leukopenia $\left(p=1.25 \times 10^{-7}\right.$; Table 4), however no statistic association was observed for late leukopenia $(p=0.20)$. Among the 23 patients of leukopenia, 10 of them developed neutropenia. NUDT15 R139C was also strongly related with the incidence of neutropenia ( $43.5 \%$ mutation vs. $0 \%$ wild type, $p=3.78 \times 10^{-4}$; OR $=12.21$; 95\% CI, 2.38-62.56). The patient with the NUDT15 R139C homozygote status (TT) and one patient with heterozygous (TC) developed severe hair loss, while no patients were observed with this phenomenon with the wild genotype (CC).

Of the 83 patients with wild type (TT) of TPMT*3C, 22 (26.5\%) suffered leukopenia; of 4 patients with heterozygous (TC), 1 patient (25.0\%) developed leukopenia. Thus, there was no significant association of TPMT*3C with AZA-induced leukopenia ( $p=0.95$; Table 3).

\section{ROC for an Additive Prediction Model of Leukopenia Using NUDT15 R139C}

We found that the NUDT15 R139C allele had a sensitivity of $84.4 \%$ and specificity of $78.3 \%$ for predicting leukopenia induced by AZA. Applying the ROC curve as an additive prediction model, the area under the curve with $95 \%$ CI $0.70-0.92$ was 0.81 . Using the NUDT15 R139C model to predict early leukopenia, the sensitivity and the specificity were $84.4,81.0 \%$, respectively, and the area under the curve with $95 \%$ CI $0.72-0.94$ was 0.83 (Figure 1).

\section{Association of 6-TGN Levels With Leukopenia}

The 6-TGN concentration of 87 patients ranged from 33.7 to $354.8 \mathrm{pmol} / 8^{*} 10^{8} \mathrm{RBC}$, mean (SD) 6-TGN concentration was 140.3 (62.38) $\mathrm{pmol} / 8^{*} 10^{8}$ RBC. The associations of concentrations of 6-TGN with different variables were shown in Supplement Table S1. Overall, the level of 6-TGN was not significantly different between patients with leukopenia or the controls $(p=0.15)$, and no significant difference was observed between patients with early or late leukopenia $(p=0.12)$. Additionally, the 6-TGN concentration was not found to have a significant association with leukopenia in different genotypes, NUDT15 R139C and TPMT*3C genotypes $(p=0.62$ and 0.25 respectively).

\section{DISCUSSION}

AZA is widely used as an immunosuppressive agent and its efficacy is endorsed by clinical studies. However, leukopenia,

TABLE 2 | Allele distribution of NUDT15 R139C and TPMT*3C genotypes.

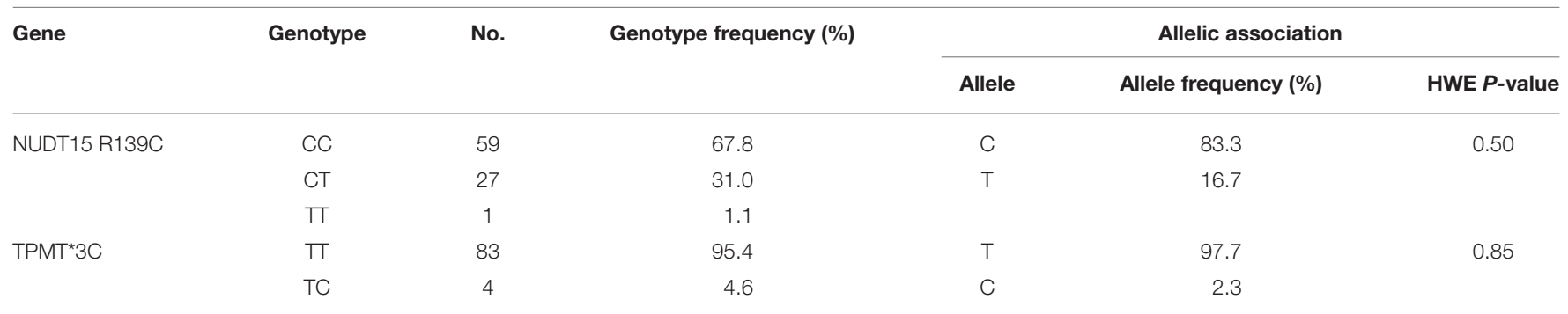

No., number of; HWE, Hardy-Weinberg equilibrium.

TABLE 3 | Association of leukopenia with NUDT15 R139C and TPMT*3C genotypes.

\begin{tabular}{|c|c|c|c|c|c|c|c|}
\hline Gene & Genotype & Prevalence of leukopenia & $p$ & \multicolumn{4}{|c|}{ Allelic association } \\
\hline & CT & $63.0 \%(17 / 27)$ & & $\mathrm{T}$ & $39.1 \%$ & & \\
\hline & $\pi$ & $100 \%(1 / 1)$ & & & & & \\
\hline TPMT³C & $\Pi$ & $26.5 \%(22 / 83)$ & 0.95 & $\mathrm{~T}$ & $97.8 \%$ & 0.95 & $1.08(0.11-10.65)$ \\
\hline
\end{tabular}

OR, odds ratio; $95 \% \mathrm{Cl}$, 95\% confidence interval. 
TABLE 4 | Association of different phase of leukopenia with NUDT15 R139C genotypes.

\begin{tabular}{|c|c|c|c|c|c|c|c|}
\hline $\begin{array}{l}\text { Gene } \\
\text { NUDT15 R139C }\end{array}$ & \multicolumn{3}{|c|}{ Genotype frequency number (\%) } & $p$ & \multicolumn{3}{|c|}{ Allelic association } \\
\hline Leukopenia & $5(8.5)$ & 17(63.0) & $1(100)$ & $1.86 \times 10^{-7}$ & $39.1 \%$ & $1.79 \times 10^{-7}$ & $7.59(3.16-18.21)$ \\
\hline Late leukopenia (>8w) & $1(1.7)$ & $1(3.7)$ & 0 & 0.20 & $25 \%$ & 0.22 & $3.93(0.37-41.39)$ \\
\hline controls & $54(91.5)$ & $10(37.0)$ & 0 & & $7.8 \%$ & & \\
\hline
\end{tabular}

OR, odds ratio; 95\% Cl, 95\% confidence interval; controls, the patients without leukopenia; 8 w, the eighth week.

\section{A ROC for an additive prediction model of leukopenia using NUDT15 R139C}

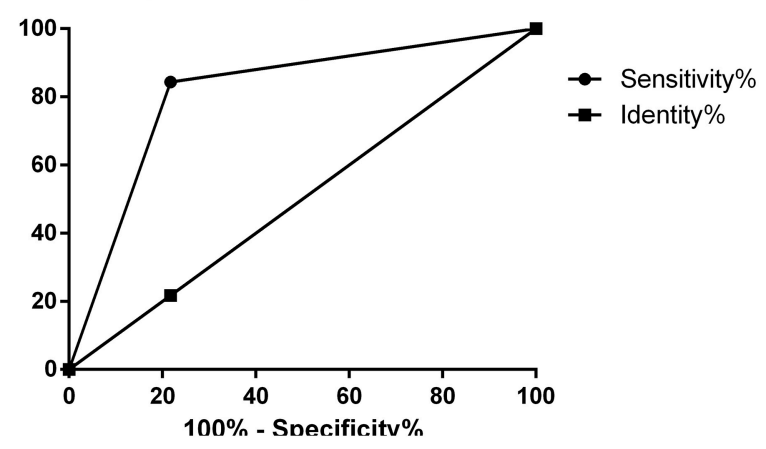

\section{B ROC for an additive prediction model of early leukopenia using NUDT15 R139C}

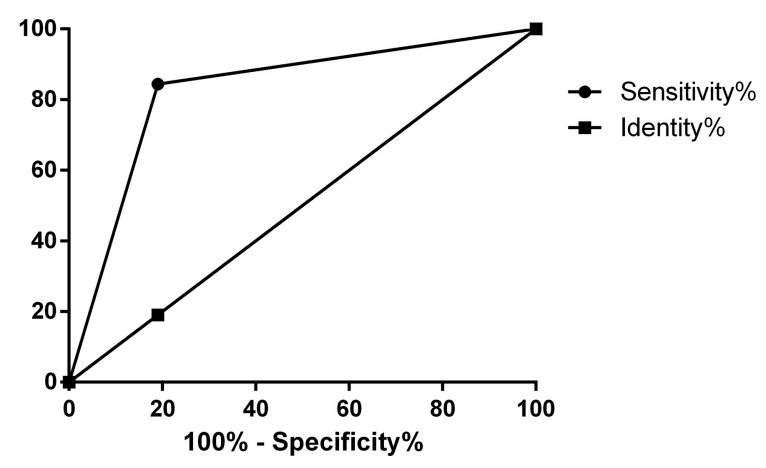

FIGURE 1 | ROC for an additive prediction model of leukopenia and early leukopenia using NUDT15 R139C. (A) ROC for an additive prediction model of leukopenia using NUDT15 R139C. (Area under the ROC curve is $0.81,95 \%$ confidence interval is $0.70-0.92$ and $p$-value $<0.0001$, the sensitivity and specificity were $84.4,78.3 \%$ respectively). (B) ROC for an additive prediction model of early leukopenia using NUDT15 R139C. (Area under the ROC curve is $0.83,95 \%$ confidence interval is $0.72-0.94$ and $p$-value $<0.0001$, the sensitivity and specificity were $84.4,81.0 \%$ respectively).

the most common ADR, still restricts its clinical application. In this study, we found a strong association of NUDT15 R139C with AZA-induced leukopenia, neutropenia and hair loss. It is an independent and first verification in Chinese autoimmune disease patients.

The incidence of leukopenia is about $5.0 \%$ based on the research on Caucasians (Ansari et al., 2008; Timmer et al., 2012), while the number is apparently higher in Asians. 35.4\% (346/978) of Korean Crohn's disease (CD) patients at a median AZA dose of $1.7 \mathrm{mg} / \mathrm{kg}$ per day suffered leukopenia (Yang et al., 2014). $25.1 \%(34 / 135)$ of Japanese IBD patients had leukopenia at the AZA dose was $0.93 \mathrm{mg} / \mathrm{kg}$ per day (Kakuta et al., 2016) and in Chinese IBD patients, $18.1 \%$ (36/199) had observed leukopenia at a median AZA does of $50 \mathrm{mg} /$ day (Gao et al., 2012). Higher frequency of severe leukopenia was observed in East-Asians, even if the AZA dose was lower than the recommended dosage.

The FDA recommend TPMT gene testing to prevent AZA-induced leukopenia before AZA treatment. TPMT plays a key role in the metabolism of AZA. Mutations cause abnormal metabolism and lead to accumulation of excess 6-TGN (Chouchana et al., 2014), which can increase the risk of AZAinduced adverse reactions, especially for leukopenia (Gearry et al., 2004). Paradoxically, AZA-induced leukopenia is more common in Asians, and compared with the $10.0 \%$ prevalence of TPMT reported in European population (Collie-Duguid et al., 1999). (Yang et al., 2014) demonstrated TPMT mutations in only $3.8 \%$ of Korean CD with leukopenia and (Zhu et al., 2016) reports only $4.6 \%$ Chinese individuals with IBD experiencing leukopenia carried variant TPMT alleles. There was only $4.6 \%$ of TPMT mutation prevalence among Chinese in our study which is similar to other reports in Asians (Ban et al., 2008; Lee et al., 2015). In our study, there were four patients $(4.6 \%)$ who carried heterozygote mutant allele of TPMT*3C and only one patient $(4.3 \%)$ with leukopenia carried TPMT variant alleles, however 22 patients (26.5\%) with $T_{P M T} 3 C$ wild type suffered leukopenia. Low mutant frequency of TPMT*3C in Asian population suggested this gene may have limited prediction value for AZA-induced leukopenia in Chinese patients. Although genetic polymorphism of TPMT has been recognized as a major risk factor for the development of AZA-induced myelotoxicity (Colombel et al., 2000), adverse events cannot be explained very well in Chinese patients by a normal or mutated TPMT genotype (Cao et al., 2009).

NUDT15 is a member of the nudix hydrolase enzyme family which mainly consists of pyrophosphohydrolases that act upon nucleoside diphosphates linked to other moieties, X (Bessman et al., 1996). (Moriyama et al., 2016) suggested that NUDT15 may prevent the incorporation of 6-thio-GTP (TGTP) and 6thio-deoxyGTP (TdGTP) into DNA by dephosphorylating the thiopurine-active metabolites TGTP and TdGTP, thus negatively affecting the desired cytotoxic effects of AZA in vivo (Sandborn et al., 2000; Roberts and Barclay, 2015; Tanaka et al., 2015; 
Asada et al., 2016; Chiengthong et al., 2016; Kakuta et al., 2016; Liang et al., 2016; Moriyama et al., 2016; Park et al., 2016; Sato et al., 2016). In-vitro studies showed a higher percentage of apoptosis and necrosis in cells transfected with the NUDT15 R139C construction compared with cells with the wild type (Yang et al., 2014). In 2014, (Yang et al., 2014) showed there was a significant association between NUDT15 R139C and AZAinduced leukopenia in Korean IBD patients $\left(p=5.58 \times 10^{-43}\right.$, $\mathrm{OR}=8.61$ ). That was also verified in Japanese IBD patients, as the NUDT15 R139C was correlated with early leukopenia $\left(p=1.92 \times 10^{-16}, \mathrm{OR}=28.4\right.$; Kakuta et al., 2016). Our result is consistent with previous studies that NUDT15 R139C was associated with early leukopenia. However, in X. Zhu's research, NUDT15 R139C was not only associated with early leukopenia $(0-8$ weeks $)\left(p=2.13 \times 10^{-19}, \mathrm{OR}=15.67\right)$ but also with middle $\left(8-24\right.$ weeks) and late ( $>24$ weeks) leukopenia $\left(p=4.51 \times 10^{-9}\right.$, $\mathrm{OR}=12.06 ; p=0.022$, OR $=3.91$ respectively) in Chinese IBD patients (Zhu et al., 2016). We enrolled 87 patients and the follow-up visiting was just 12 weeks which may limit our clinical results. Further studies using larger samples and longer followup visiting are needed to replicate our findings. Additionally, we also found that NUDT15 R139C was correlated with neutropenia $\left(p=3.78 \times 10^{-4} ; \mathrm{OR}=12.21\right)$. (Kim et al., 2017) reported that the sensitivity was $85.7 \%$ and specificity was $92.2 \%$ by an additive prediction model of early leukopenia using NUDT15 R139C in Korean neurological diseases In our study, we calculated the predictability of NUDT15 variant allele for early leukopenia and leukopenia respectively. The result showed that NUDT15 R139C variants predicted early leukopenia better (the sensitivity and the specificity were $84.4,81.0 \%$, respectively).

In addition, such association has been replicated in multiple independent follow-up studies and also demonstrated ethnic diversity. The NUDT15 R139C mutant allele frequency is high in Asians and Hispanics (about 10\% in Asians), low in Caucasians (about 0.2\%) and not found in Africans (Yang et al., 2015). NUDT15 R139C mutant frequency were 10.4 and $12 \%$ in Korean and Japanese IBD patients, respectively. In this study, we found that the frequency in Chinese autoimmune disease patients was about $32.1 \%$ which was higher than previous reports. The percentage of females was much higher than males (90.8 vs. 9.2\%) in our study since females are more susceptible to autoimmune diseases like SLE, SS. We inferred the mutant frequency of NUDT15 R139C may be higher in female patients than males, but this hypothesis needs to be validated in further large sample studies. These studies, together with our data, suggest NUDT15 R139C may have a greater prediction ability than $T P M T^{*} 3 C$ genotyping for prospective risk assessment of AZA-induced leukopenia in Asian populations.

It is well-known that 6-TGN are the predominant active metabolites of AZA, and the accumulation of 6-TGN can induce adverse reactions, particularly for leukopenia (Armstrong et al., 2011). Monitoring 6-TGN blood concentrations routinely in patients receiving AZA has also been recommended (Dubinsky et al., 2000; Cuffari et al., 2004; Wright et al., 2004). The (Zhu et al., 2016) study suggested there was a slight difference in 6-TGN concentration between patients with or without leukopenia ( $p=0.067$ ), while 6-TGN levels were significantly correlated with leukopenia in the patients of NUDT15 wild type $(p=0.0055)$. However, (Asada et al., 2016) reported there was no significant 6-TGN level difference in 161 Japanese IBD patients for NUDT15 genotypes. In our research, we took the same measurement method of 6-TGN with previous studies in IBD, and no statistically significant concentration difference was observed between different NUDT15 genotypes ( $p=0.62$ ). Among our 87 Chinese autoimmune disease patients, the 6-TGN levels ranged from 33.7 to $354.8 \mathrm{pmol} / 8^{*} 10^{8} \mathrm{RBC}$, mean (SD) was 140.3 (62.38) $\mathrm{pmol} / 8^{*} 10^{8} \mathrm{RBC}$, which was significantly lower than the target 6-TGN monitoring range, 235-450 pmol $/ 8^{*} 10^{8}$ RBC for IBD (Osterman et al., 2006). In patients without leukopenia, only three of them had 6TGN levels within the recommend range (3/64), whose 6TGN concentrations were 254.6, 277.2, $354.8 \mathrm{pmol} / 8^{*} 10^{8} \mathrm{RBC}$, respectively. There were also three $(3 / 23)$ within the IBD range $\left(240,289.9,347.5 \mathrm{pmol} / 8^{*} 10^{8} \mathrm{RBC}\right.$, respectively) of patients with leukopenia. None of our patients were beyond the recommended 6-TGN monitoring range established for IBD. It indicates that the monitoring range for autoimmune disease patients needs to be established in the future. In addition (Moriyama et al., 2016), suggested that NUDT15 R139C may dephosphorylate the AZA-active metabolites TGTP and TdGTP rather than 6-TGN, which may explain why our concentrations were lower than the established range for IBD. The new therapeutic monitoring method detecting the metabolites TGTP and TdGTP may have more clinical value than 6-TGN.

In conclusion, we replicated previous findings that the NUDT15 R139C variant is a potential predictor for AZAinduced leukopenia in Chinese, extended this finding to patients with various autoimmune diseases and identified its specific association with leukopenia, especially for early leukopenia and neutropenia. Moreover, we found that the concentration of 6TGN in autoimmune disease patients was much lower than the recommended range for IBD patients, suggesting a need for establishment of a new monitoring range for autoimmune disease patients. NUDT15 R139C gene testing may offer a more useful and effective way than TPMT to predict the AZA-induced leukopenia for physicians. They will promote the optimization of AZA medication in autoimmune disease patients.

\section{ETHICS STATEMENT}

This study was carried out in accordance with the recommendations of the ethical guidelines and approved by the research ethics committee of Drum Tower Hospital affiliated Nanjing University Medical School, Nanjing, China (2017-101-01) with written informed consent from all subjects. All subjects gave written informed consent in accordance with the Declaration of Helsinki. The protocol was approved by the ethical guidelines and approved by the research ethics committee of Drum Tower Hospital affiliated Nanjing University Medical School, Nanjing, China (2017-101-01). 


\section{AUTHOR CONTRIBUTIONS}

$\mathrm{XF}$ and QS: wrote the article; XF, QS, HZ, BH, SW, YF, and WG: designed the research; XF, HZ, BH, SW, and LG: performed the research; XF, and QS: analyzed the data.

\section{ACKNOWLEDGMENTS}

We gratefully acknowledge Ruijin Cui for statistical support, Zhenhan Zhu, Shuyin Zhang, and Zhi Cao for technical

\section{REFERENCES}

Ansari, A., Arenas, M., Greenfield, S. M., Morris, D., Lindsay, J., Gilshenan, K., et al. (2008). Prospective evaluation of the pharmacogenetics of Azathioprine in the treatment of inflammatory bowel disease. Aliment. Pharmacol. Ther. 28, 973-983. doi: 10.1111/j.1365-2036.2008.03788.x

Armstrong, L., Sharif, J. A., Galloway, P., McGrogan, P., Bishop, J., and Russell, R. K. (2011). Evaluating the use of metabolite measurement in children receiving treatment with a thiopurine. Aliment. Pharmacol. Ther. 34, 1106-1114. doi: 10.1111/j.1365-2036.2011.04848.x

Asada, A., Nishida, A., Shioya, M., Imaeda, H., Inatomi, O., Bamba, S., et al. (2016). NUDT15 R139C-related thiopurine leukocytopenia is mediated by 6-thioguanine nucleotide-independent mechanism in Japanese patients with inflammatory bowel disease. J. Gastroenterol. 51, 22-29. doi: 10.1007/s00535-015-1142-4

Ban, H., Andoh, A., Tanaka, A., Tsujikawa, T., Sasaki, M., Saito, Y., et al. (2008). Analysis of thiopurine S-methyltransferase genotypes in Japanese patients with inflammatory bowel disease. Intern. Med. 47, 1645-1648. doi: 10.2169/internalmedicine.47.1268

Bertsias, G., Ioannidis, J. P., Boletis, J., Bombardieri, S., Cervera, R., Dostal, C., et al. (2008). EULAR recommendations for the management of systemic lupus erythematosus. Report of a Task Force of the EULAR Standing Committee for International Clinical Studies Including Therapeutics. Ann. Rheum. Dis. 67, 195-205. doi: 10.1136/ard.2007.070367

Bessman, M. J., Frick, D. N., and O’Handley, S. F., Bessman, M. J., Frick, D. N., and O'Handley, S. F. (1996). The MutT proteins or "Nudix" hydrolases, a family of versatile, widely distributed, "housecleaning" enzymes. J. Biol. Chem. 271, 25059-25062. doi: 10.1074/jbc.271.41.25059

Boonsrirat, U., Angsuthum, S., Vannaprasaht, S., Kongpunvijit, J., Hirankarn, N., Tassaneeyakul, W., et al. (2008). Azathioprine-induced fatal myelosuppression in systemic lupus erythematosus patient carrying TPMT*3C polymorphism. Lupus 17, 132-134. doi: 10.1177/0961203307085255

Cao, Q., Zhu, Q., Shang, Y., Gao, M., and Si, J. (2009). Thiopurine methyltransferase gene polymorphisms in Chinese patients with inflammatory bowel disease. Digestion 79, 58-63. doi: 10.1159/000205268

Carter, M., Jemth, A. S., Hagenkort, A., Page, B. D., Gustafsson, R., Griese, J. J., et al. (2015). Crystal structure, biochemical and cellular activities demonstrate separate functions of MTH1 and MTH2. Nat. Commun. 6:7871. doi: $10.1038 /$ ncomms 8871

Chiengthong, K., Ittiwut, C., Muensri, S., Sophonphan, J., Sosothikul, D., Seksan, P., et al. (2016). NUDT15c.415C $>$ T increases risk of 6mercaptopurine induced myelosuppression during maintenance therapy in children with acute lymphoblastic leukemia. Haematologica 101, e24-e26. doi: 10.3324/haematol.2015.134775

Chouchana, L., Narjoz, C., Roche, D., Golmard, J. L., Pineau, B., Chatellier, G., et al. (2014). Interindividual variability in TPMT enzyme activity: 10 years of experience with thiopurine pharmacogenetics and therapeutic drug monitoring. Pharmacogenomics 15, 745-757. doi: 10.2217/pgs.14.32

Collie-Duguid, E. S., Pritchard, S. C., Powrie, R. H., Sludden, J., Collier, D. A., Li, T., et al. (1999). The frequency and distribution of thiopurine methyltransferase alleles in Caucasian and Asian populations. Pharmacogenetics 9, 37-42. doi: 10.1097/00008571-199902000-00006 assistance. This work was supported by grants from the Nanjing Medical Science and Technique Development Foundation (QRX17140), Jiangsu Provincial Medical Innovation Team and Fundamental Research Funds for the Central Universities (021414380201).

\section{SUPPLEMENTARY MATERIAL}

The Supplementary Material for this article can be found online at: https://www.frontiersin.org/articles/10.3389/fphar. 2018.00460/full\#supplementary-material

Colombel, J. F., Ferrari, N., Debuysere, H., Marteau, P., Gendre, J. P., Bonaz, B., et al. (2000). Genotypic analysis of thiopurine S-methyltransferase in patients with Crohn's disease and severe myelosuppression during Azathioprine therapy. Gastroenterology 118, 1025-1030. doi: 10.1016/S0016-5085(00)70354-4

Connell, W. R., Kamm, M. A., Ritchie, J. K., and Lennard-Jones, J. E. (1993). Bone marrow toxicity caused by Azathioprine in inflammatory bowel disease: 27 years of experience. Gut 34, 1081-1085. doi: 10.1136/gut.34.8.1081

Cuffari, C., Dassopoulos, T., Turnbough, L., Thompson, R. E., and Bayless, T. M. (2004). Thiopurine methyltransferase activity influences clinical response to Azathioprine in inflammatory bowel disease. Clin. Gastroenterol. Hepatol. 2, 410-417. doi: 10.1016/S1542-3565(04)00127-2

Cuffari, C., Théorêt, Y., Latour, S., and Seidman, G. (1996). 6-Mercaptopurine metabolism in Crohn's disease: correlation with efficacy and toxicity. Gut 39, 401-406. doi: 10.1136/gut.39.3.401

de Jong, D. J., Derijks, L. J., Naber, A. H., Hooymans, P. M., and Mulder, C. J. (2003). Safety of thiopurines in the treatment of inflammatory bowel disease. Scand. J. Gastroenterol. Suppl. 69-72. doi: 10.1080/00855920310002726

Dervieux, T., and Boulieu, R. (1998). Simultaneous determination of 6thioguanine and methyl 6-mercaptopurine nucleotides of Azathioprine in red blood cells by HPLC. Clin. Chem. 44, 551-555.

Dubinsky, M. C., Lamothe, S., Yang, H. Y., Targan, S. R., Sinnett, D., Théorêt, Y., et al. (2000). Pharmacogenomics and metabolite measurement for 6mercaptopurine therapy in inflammatory bowel disease. Gastroenterology 118, 705-713. doi: 10.1016/S0016-5085(00)70140-5

Fangbin, Z., Xiang, G., Minhu, C., Liang, D., Feng, X., Min, H., et al. (2012). Should thiopurine methyltransferase genotypes and phenotypes be measured before thiopurine therapy in patients with inflammatory bowel disease? Ther. Drug Monit. 34, 695-701 doi: 10.1097/FTD.0b013e3182731925

Gao, X., Zhang, F. B., Ding, L., Liu, H., Wang, X. D., Chen, B. L., et al. (2012). The potential influence of 5-aminosalicylic acid on the induction of myelotoxicity during thiopurine therapy in inflammatory bowel disease patients. Eur. J. Gastroenterol. Hepatol. 24, 958-964. doi: 10.1097/MEG.0b013e3283545ae3

Gearry, R. B., Barclay, M. L., Burt, M. J., Collett, J. A., and Chapman, B. A. (2004). Thiopurine drug adverse effects in a population of New Zealand patients with inflammatory bowel disease. Pharmacoepidemiol. Drug Saf. 13, 563-567. doi: 10.1002 pds.926

Hanai, H., Iida, T., Takeuchi, K., Arai, O., Watanabe, F., Abe, J., et al. (2010). Thiopurine maintenance therapy for ulcerative colitis: the clinical significance of monitoring 6-thioguanine nucleotide. Inflamm. Bowel Dis. 16, 1376-1381. doi: 10.1002/ibd.21190

Hiratsuka, M., Inoue, T., Omori, F., Agatsuma, Y., and Mizugaki, M. (2000). Genetic analysis of thiopurine methyltransferase polymorphism in a Japanese population. Mutat. Res. 448, 91-95. doi: 10.1016/S0027-5107(00)00 004-X

Janssens, P., Arnaud, L., Galicier, L., Mathian, A., Hie, M., Sene, D., et al. (2013). Lupus enteritis: from clinical findings to therapeutic management. Orphanet $J$. Rare Dis. 8:67. doi: 10.1186/1750-1172-8-67

Jharap, B., Seinen, M. L., de Boer, N. K., van Ginkel, J. R., Linskens, R. K., Kneppelhout, J. C., et al. (2010). Thiopurine therapy in inflammatory bowel disease patients: analyses of two 8-year intercept cohorts. Inflamm. Bowel Dis. 16, 1541-1549. doi: 10.1002/ibd.21221 
Kakuta, Y., Naito, T., Onodera, M., Kuroha, M., Kimura, T., Shiga, H., et al. (2016). NUDT15 R139C causes thiopurine-induced early severe hair loss and leukopenia in Japanese patients with IBD. Pharmacogenomics J. 16, 280-285. doi: 10.1038/tpj.2015.43

Kim, S. Y., Shin, J. H., Park, J. S., Kang, S. Y., Nam, T. S., Kim, J. K., et al. (2017). NUDT15 p.R139C variant is common and strongly associated with Azathioprine-induced early leukopenia and severe alopecia in Korean patients with various neurological diseases. J. Neurol. Sci. 378, 64-68. doi: 10.1016/j.jns.2017.04.041

Lee, K. M., Kim, Y. S., Seo, G. S., Kim, T. O., Yang, S. K., and IBD Study Group of the Korean Association for the Study of Intestinal Diseases. (2015). Use of thiopurines in inflammatory bowel disease: a consensus statement by the Korean association for the study of intestinal diseases (KASID). Intest. Res. 13, 193-207. doi: 10.5217/ir.2015.13.3.193

Liang, D. C., Yang, C. P., Liu, H. C., Jaing, T. H., Chen, S. H., Hung, I. J., et al. (2016). NUDT15 gene polymorphism related to mercaptopurine intolerance in Taiwan Chinese children with acute lymphoblastic leukemia. Pharmacogenomics J. 16, 536-539. doi: 10.1038/tpj.2015.75

Moon, W., Loftus, E. V. Jr. (2016). Review article: recent advances in pharmacogenetics and pharmacokinetics for safe and effective thiopurine therapy in inflammatory bowel disease. Aliment. Pharmacol. Ther. 43, 863-883. doi: 10.1111/apt.13559

Moriyama, T., Nishii, R., Perez-Andreu, V., Yang, W., Klussmann, F. A., Zhao, X., et al. (2016). NUDT15 polymorphisms alter thiopurine metabolism and hematopoietic toxicity. Nat. Genet. 48, 367-373. doi: 10.1038/ng.3508

Ngo, S., Sauvetre, G., Vittecoq, O., Lévesque, H., and Marie, I. (2011). Azathioprine-associated severe myelosuppression: indication of routine determination of thiopurine S-methyltransferase variant?. La Revue de Medecine Interne 32, 373-376. doi: 10.1016/j.revmed.2010.09.006

Okon, L. G., and Werth, V. P. (2013). Cutaneous lupus erythematosus: diagnosis and treatment. Best Pract. Res. Clin. Rheumatol. 27, 391-404. doi: 10.1016/j.berh.2013.07.008

Osterman, M. T., Kundu, R., Lichtenstein, G. R., and Lewis, J. D. (2006). Association of 6-thioguanine nucleotide levels and inflammatory bowel disease activity: a meta-analysis. Gastroenterology 130, 1047-1053 doi: 10.1053/j.gastro.2006.01.046

Park, S. K., Hong, M., Ye, B. D., Kim, K. J., Park, S. H., Yang, D. H., et al. (2016). Influences of XDH genotype by gene-gene interactions with SUCLA2 for thiopurine-induced leukopenia in Korean patients with Crohn's disease. Scand. J. Gastroenterol. 51, 684-691. doi: 10.3109/00365521.2015.1133698

Ramos-Casals, M., Tzioufas, A. G., Stone, J. H., Sisó, A., and Bosch, X. (2010). Treatment of primary Sjogren syndrome: a systematic review. JAMA 304, 452-460. doi: 10.1001/jama.2010.1014

Relling, M. V., Gardner, E. E., Sandborn, W. J., Schmiegelow, K., Pui, C. H., Yee, S. W., et al. (2013). Clinical pharmacogenetics implementation consortium guidelines for thiopurine methyltransferase genotype and thiopurine dosing: 2013 update. Clin. Pharmacol. Ther. 93, 324-325. doi: 10.1038/clpt.2013.4

Roberts, R. L., and Barclay, M. L. (2015). Update on thiopurine pharmacogenetics in inflammatory bowel disease. Pharmacogenomics 16, 891-903. doi: $10.2217 /$ pgs. 15.29

Sandborn, W., Sutherland, L., Pearson, D., May, G., Modigliani, R., and Prantera, C. (2000). Azathioprine or 6-mercaptopurine for inducing remission of Crohn's disease. Cochrane Database Syst. Rev. CD000545 doi: 10.1002/14651858.CD000545
Sato, M., Harada, M., Oishi, H., Wada-Hiraike, O., Hirata, T., Nagasaka, K., et al. (2016). Vaginal stenposis after gonadotropin-releasing hormone agonist therapy during treatment for acute lymphoblastic leukemia. J. Low. Genit. Tract Dis. 20, e11-e13. doi: 10.1097/LGT.0000000000000175

Somerville, L., Krynetski, E. Y., Krynetskaia, N. F., Beger, R. D., Zhang, W., Marhefka, C. A., et al. (2003). Structure and dynamics of thioguanine-modified duplex DNA. J. Biol. Chem. 278, 1005-1011. doi: 10.1074/jbc.M204243200

Tanaka, Y., Kato, M., Hasegawa, D., Urayama, K. Y., Nakadate, H., Kondoh, K., et al. (2015). Susceptibility to 6-MP toxicity conferred by a NUDT15 variant in Japanese children with acute lymphoblastic leukaemia. Br. J. Haematol. 171, 109-115. doi: 10.1111/bjh.13518

Teml, A., Schaeffeler, E., Herrlinger, K. R., Klotz, U., and Schwab, M. (2007). Thiopurine treatment in inflammatory bowel disease: clinical pharmacology and implication of pharmacogenetically guided dosing. Clin. Pharmacokinet. 46, 187-208. doi: 10.2165/00003088-200746030-00001

Timmer, A., McDonald, J. W., Tsoulis, D. J., and Macdonald, J. K. (2012). Azathioprine and 6-mercaptopurine for maintenance of remission in ulcerative colitis. Cochrane Database Syst. Rev. CD000478. doi: 10.1002/14651858.CD000478.pub3

Timmer, A., Patton, P. H., Chande, N., McDonald, J. W., and MacDonald, J. K. (2016). Azathioprine and 6-mercaptopurine for maintenance of remission in ulcerative colitis. Cochrane Database Syst. Rev. CD000478. doi: 10.1002/14651858.CD000478.pub4

Wright, S., Sanders, D. S., Lobo, A. J., and Lennard, L. (2004). Clinical significance of Azathioprine active metabolite concentrations in inflammatory bowel disease. Gut 53, 1123-1128. doi: 10.1136/gut.2003.032896

Yang, J. J., Landier, W., Yang, W., Liu, C., Hageman, L., Cheng, C., et al. (2015). Inherited NUDT15 variant is a genetic determinant of mercaptopurine intolerance in children with acute lymphoblastic leukemia. J. Clin. Oncol. 33, 1235-1242. doi: 10.1200/JCO.2014.59.4671

Yang, S. K., Hong, M., Baek, J., Choi, H., Zhao, W., Jung, Y., et al. (2014). A common missense variant in NUDT15 confers susceptibility to thiopurineinduced leukopenia. Nat. Genet. 46, 1017-1020. doi: 10.1038/ng.3060

Zhu, Q., and Cao, Q. (2012). Thiopurine methyltransferase gene polymorphisms and activity in Chinese patients with inflammatory bowel disease treated with Azathioprine. Chin. Med. J. 125, 3665-3670. doi: 10.3760/cma.j.issn.0366-6999.2012.20.012

Zhu, X., Wang, X. D., Chao, K., Zhi, M., Zheng, H., Ruan, H. L., et al. (2016). NUDT15 polymorphisms are better than thiopurine S-methyltransferase as predictor of risk for thiopurine-induced leukopenia in Chinese patients with Crohn's disease. Aliment. Pharmacol. Ther. 44, 967-975. doi: 10.1111/apt. 13796

Conflict of Interest Statement: The authors declare that the research was conducted in the absence of any commercial or financial relationships that could be construed as a potential conflict of interest.

Copyright (c) 2018 Fei, Shu, Zhu, Hua, Wang, Guo, Fang and Ge. This is an openaccess article distributed under the terms of the Creative Commons Attribution License (CC BY). The use, distribution or reproduction in other forums is permitted, provided the original author(s) and the copyright owner are credited and that the original publication in this journal is cited, in accordance with accepted academic practice. No use, distribution or reproduction is permitted which does not comply with these terms. 